\title{
Optimal Control Model for Attack of Worms in Wireless Sensor Network
}

\author{
Neha Keshri and Bimal Kumar Mishra ${ }^{a}$ \\ Department of Applied Mathematics, Birla Institute of Technology, Mesra, Ranchi, \\ India - 835215 \\ drbimalmishra@gmail.com (Bimal Kumar Mishra),keshri.neha7@gmail.com (Neha \\ Keshri)
}

\begin{abstract}
Wireless Sensor Network (WSN) is composed of large number of sensor nodes which communicate with each other through wireless medium. Such networks are prone to the attack of self-replicating worms known as malware used to bring different form of attacks on the WSN during the communication of the sensor nodes. Efficient countermeasures to anticipate malware hazards are to be developed and understand the threats they pose before they emerge in the hands of the attackers. In this paper, we propose security mechanisms using three different epidemic models with proper countermeasure which depends on the incidence of worm infections. A special crashed compartment is introduced that includes the nodes which crash out due to induced infection or reason other than the attack. The crashed class is expected to provide a new view-point which can help in optimizing the level of infection and of corresponding recovery of sensor nodes in the wireless sensor network. The past and present information of the worms represented in the form of Information variable and a Control function to minimize the attack of malware in WSN is taken into account in the different epidemic models developed. Further threshold values have been obtained that determine whether the worms completely die out or not. The stability of the worms-free and endemic equilibrium of the epidemic models is well discussed and it is also shown that the models may undergo a forward bifurcation. To validate the models extensive simulation is carried out. The results may help in developing a proper secure mechanism in WSN.
\end{abstract}

Keywords: Epidemic models; Worms; Wireless sensor network; Global stability; endemic equilibrium; optimal control

\section{Introduction}

Wireless sensor network is prone to the propagation of self-replicating malicious signals known as malware, which are used to bring different form of attacks on the wireless network. The attack can be of lesser intrusive in nature (such as violation of confidentiality or privacy, as in traffic analysis and eves dropping) as well as of higher intrusive in nature (such as disrupt the normal functions of the sensor nodes or alter the network traffic and hence destroy the integrity of the information). The first step in devising efficient countermeasures is to anticipate malware hazards and understand the threats they pose before they emerge in the hands of the attackers. Specific attacks such

\footnotetext{
${ }^{\mathrm{a}}$ Corresponding author

Email: drbimalmishra@gmail.com (Bimal Kumar Mishra), keshri.neha7@gmail.com (Neha Keshri)

Mobile number: +91-9430764860, FAX: +91-651-2276183
} 
as the wormhole, Sinkhole and Sybil that utilize vulnerabilities in the routing protocols in the wireless sensor network and their countermeasures have been investigated before they were actually launched [1].The attacks of the malicious signals in the wireless sensor network are epidemic in nature. Malicious signals targeting wireless devices, for example, the Cabir worm, can repeatedly send itself to Bluetooth enable devices inside it's host's scanning range. Similarly, the Mabir worm uses scanning techniques to attack. The spreading behavior of the Mabir and Cabir worm are epidemic in nature [2]. Thus, to defend the sensor nodes against these types of malware attacks, we propose security mechanism using epidemic models.

Biological epidemic models will be helpful to understand the dynamical behavior of the malware attack in Wireless sensor network (WSN). Global stability of SIR epidemic model with distributed delay have been studied by Beretta et.al in which the time for an individual to lose infectiousness is well discussed [3]. Song and Cheng have proposed the effect of time delay on the stability of the endemic equilibrium [4]. For all delays, some conditions were considered for the asymptotical stability of endemic equilibrium. Mishra and Jha proposed a susceptible-exposed-infectious-quarantined-removed (SEIQRS) e-epidemic model to understand the spreading behavior of worms in computer network and to reduce the infectiousness among the nodes in the computer network, a quarantine class $(\mathrm{Q})$ was introduced where the nodes which are highly infectious were forcibly isolated and kept in the class Q [5] . Ren and Yang proposed a computer virus propagation model in network with shelter which depends on the incidence of virus infection and also to optimal shelter coverage, a control problem relative to their model is taken into account [6].

As we have stated above that in wireless sensor network, autonomous devices are spatially scattered that means sensor nodes are spread randomly in the network field. Sensor nodes receive the information from the network but due to limited power, modern sensor hardware is usually designed with a low-power sleep mode. The nodes periodically put themselves into sleep mode for a certain length of time, and then after certain interval of time return to the active mode. In this way, significant energy savings can be achieved while maintaining network connectivity. Due to the limited transmission range, data generated from sensors that are far away from the sink must be relayed through intermediate nodes; i.e., a source node sends its data to its neighbor nodes, which in turn send the data to their respective neighbors. Through internet sink sends the collection of data to the main server or user. The structural representation of wireless sensor network is depicted in figure 1 .

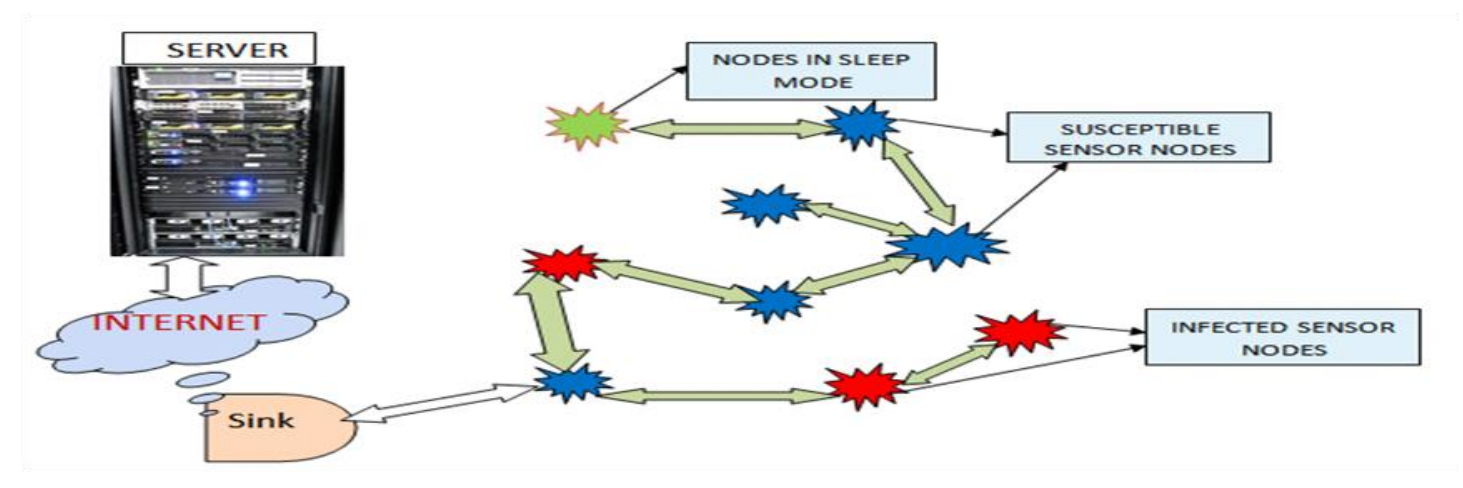

Figure 1. Structural Representation of Wireless Sensor Network 
In this paper, we propose Susceptible- Infectious-Recovered-Crashed (SIRC), Susceptible- Infectious- Quarantined- Recovered- Crashed (SIQRC) and SusceptibleInfectious-Recovered-Vaccinated-Crashed (SIRVC) models with proper countermeasure which depends on the incidence of worm infections, i.e., to what extent the proper countermeasure provides protection to the sensor nodes and also to what extent countermeasure depends on both past and current incidence of worms' infections. The SIRC model allows us to extend the classical SIR model, by considering a special compartment that includes the nodes which crash out because of infection induced or natural reasons. The crashed class is expected to provide a new view-point which can help in optimizing the level of infection and of corresponding recovery of sensor nodes in the system. In the SIQRC model, we introduce quarantining of infected nodes as a further means of enhancing the recovery of infected sensor nodes. The process of quarantining a section of infected sensor nodes is a useful measure that reduces the effective rate at which the infected number of nodes increases. The SIRVC model explores another aspect of control of the infection, which is provided by the vaccination of susceptible nodes. This method also provides us a way of controlling the effective rate of infection of new sensor nodes, by providing immunity to uninfected sensor nodes from the infection. We have made the following contributions:

(a) Provide threshold value that determines whether the worms completely die out and also study the stability of the worm's free and endemic equilibrium of the above models

(b) Shows that the models may undergo a forward bifurcation.

(c) To achieve optimal proper countermeasure, a control problem relative to our models are considered.

(d) Some numerical discussions \& extensive simulation by using MATLAB to support our mathematical conclusions.

\begin{tabular}{|ll|}
\hline & \multicolumn{1}{c|}{ Nomenclature } \\
$\mathrm{S}(\mathrm{t})$ & The susceptible population size \\
$\mathrm{I}(\mathrm{t})$ & Population size of infectious class \\
$\mathrm{R}(\mathrm{t})$ & The recovered population size \\
$\mathrm{C}(\mathrm{t})$ & Population size of crashed class \\
$\mathrm{Q}(\mathrm{t})$ & Population size of quarantined class \\
$\mathrm{V}(\mathrm{t})$ & Population size of vaccinated class \\
$\mathrm{N}(\mathrm{t})$ & The total population size \\
$\mathrm{b}$ & Birth rate (i.e., rate of addition of new sensor nodes to the network) \\
$\beta$ & Infectivity contact rate \\
$\mu$ & The per capita death rate due to reasons other than the infection \\
$\eta$ & Crashing rate due to infection \\
$\gamma$ & Rate of recovery of infected nodes \\
$\delta$ & Rate of quarantining of infected nodes \\
$\varepsilon$ & Rate of recovery of quarantined nodes \\
\hline
\end{tabular}




\section{The Susceptible- Infectious-Recovered-Crashed (SIRC) Model:}

Malware spread through the data transmission from the infectious sensor nodes to the susceptible nodes, which are vulnerable but not yet infected. Once they are vulnerable, they move into the infected compartment, the time spent by the sensor nodes in the infected compartment is the infectious period. We provide countermeasures by installing security patches that either heal the infective of the infection and render them, robust against future attacks, or immunize susceptible nodes against future attacks by rectifying their vulnerability after which they enter in the recovered compartment. We use the term recovered for the infective nodes as well as susceptible nodes which receive the security patch. Target of the attacker is to infect as many sensor nodes as they can and use the worms to disrupt the host as well as the function of the network. The worm can kill an infective host, i.e., render them completely dysfunctional- such sensor nodes are classified in crashed class. Some of the sensor nodes enter the crash compartment from the susceptible, infective or recovered compartment due to some hardware or software failure (said to be the natural death). The flow of worms in sensor network is depicted in Figure 2.

$\mathrm{S}(\mathrm{t}), \mathrm{I}(\mathrm{t}), \mathrm{R}(\mathrm{t}), \mathrm{C}(\mathrm{t})$ represents the susceptible nodes, infectious nodes, recovered nodes, and crashed nodes at any time $t$ which will be refereed as $S, I, R, C$ respectively unless and until specified in the WSN consisting of total $\mathrm{N}$ number of sensor nodes at any time $\mathrm{t}$. We further assume that, $\mathrm{S}+\mathrm{I}+\mathrm{R}=\mathrm{N}$, where the dead sensor nodes are not considered while finding the total population. As per our assumptions and the flow of worms in sensor network, depicted in figure 2, we have the following system of differential equations:

$$
\begin{array}{ll}
\frac{d S}{d t}=b-\beta S I-\mu S ; & \frac{d I}{d t}=\beta S I-(\mu+\eta+\gamma) I \\
\frac{d R}{d t}=\gamma I-\mu R ; & \frac{d C}{d t}=\mu S+\mu R+(\mu+\eta) I
\end{array}
$$

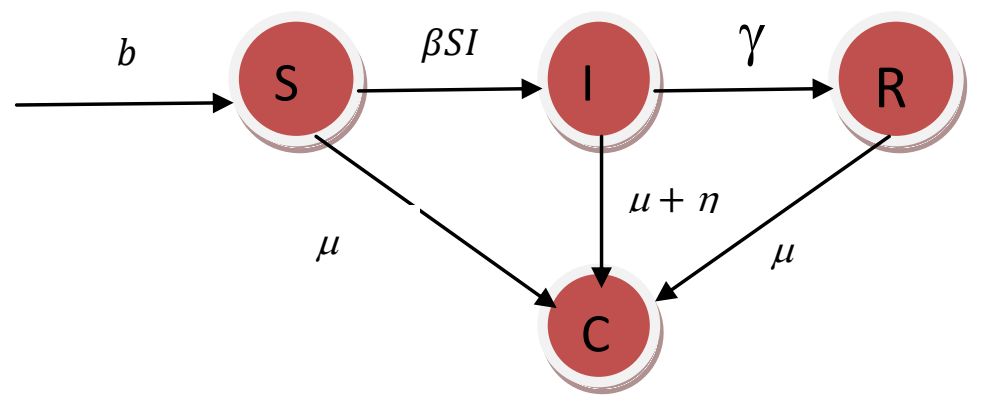

Figure 2. Schematic Representation of SIRC Model

The total population size $\mathrm{N}(\mathrm{t})$ is variable with $\frac{d N}{d t}=b-\mu N-\eta I$. In the absence of the attack of malicious signals, the population size of the node $\mathrm{N}$ approaches the 
carrying capacity $\frac{b}{\mu}$. The differential equation for $\mathrm{N}$ implies that solution of (1) starting in the positive orthan $R_{3}^{+}$approach, enter or remain in the epidemiologically meaningful subset.

$$
D_{1}=\left\{(S, I, R) / S \geq 0, I \geq 0, R \geq 0, S+I+R \leq \frac{b}{\mu}\right\}
$$

Thus, it suffices to consider solutions in region $\mathrm{D}_{1}$. Solution of the initial value problem starting in $\mathrm{D}_{1}$ and defined by (1) exists and is unique on maximal interval [7]. Since solution remain bounded in the positively invariant region $\mathrm{D}_{1}$, the maximal interval is $(0, \infty)$. Thus, initial value problem is well posed both mathematically and epidemiologically.

If we take the proper countermeasure into account and make one assumption that the antivirus programs are fully effective, then the system (1) can be rewritten as

$$
\begin{array}{llrl}
\frac{d S}{d t} & =b-\beta S I-\mu S-\alpha S ; & \frac{d I}{d t} & =\beta S I-(\mu+\eta+\gamma) I \\
\frac{d R}{d t} & =\gamma I-\mu R+\alpha S ; & \frac{d C}{d t} & =\mu(S+R)+(\mu+\eta) I
\end{array}
$$

where, $\alpha$ is the protection coverage rate of the susceptible sensor nodes and it is a critical factor in determining the degree of protection coverage. Furthermore, it is assumed that $\alpha$ is directly proportional to the incidence of worm infection, i.e., higher the incidence of worm infection, higher is the protection coverage.

Thus, $\alpha=u P$

where ' $u$ ' is a positive coefficient while $P$ is addressed to be information variable $[8,9]$. Here, the variable $\mathrm{P}$ provides the information about both the current and past state of worm infection. Thus, $\mathrm{P}$ depends on current and past values of state variable and so

$$
P=\int_{-\infty}^{t} g(S(\tau), I(\tau)) \kappa(t-\tau) d \tau
$$

where $\kappa$ is the delaying kernel [10] and $\tau$ is the distributed delay, i.e., susceptible nodes ' $\mathrm{S}$ ' and infectious nodes ' $\mathrm{I}$ ' are affected at time $\mathrm{t}$ by the state variable $\mathrm{S}$ and I at possibly all previous time $\tau$. As discussed above, the shelter coverage depends on both past and current incidence of worm infection, and so, mathematically it gives $\mathrm{g}(\mathrm{S}, \mathrm{I})=\beta \mathrm{SI}$

We have, $\kappa_{\kappa}(t-\tau)=\frac{\exp \left(\frac{-(t-\tau)}{T}\right)}{T}$, where $\mathrm{T}$ is a positive constant indicating the average delay of the collected information on the worm infection[11]. Thus from the above consideration, the model (2) can be reformulated as the following model of the wireless sensor network worm propagation, 


$$
\begin{array}{rlrl}
\frac{d S}{d t}=b-\beta S I-\mu S-u P S ; & \frac{d I}{d t}=\beta S I-(\mu+\eta+\gamma) I \\
\frac{d R}{d t}=\gamma I-\mu R+u P S & ; & \frac{d C}{d t}=\mu(S+R)+(\mu+\eta) I \\
\frac{d P}{d t}=\frac{1}{T}(\beta S I-P) &
\end{array}
$$

The system (5) can be reduced to the equivalent system

$$
\frac{d S}{d t}=b-\beta S I-\mu S-u P S ; \quad \frac{d I}{d t}=\beta S I-(\mu+\eta+\gamma) I \quad ; \quad \frac{d P}{d t}=\frac{1}{T}(\beta S I-P)
$$

On the closed positive invariant set $D=\{(S, I, P) / S \geq 0, I \geq 0, P \geq 0, S+I+P \leq b / \mu\}$ System (6) has two possible equilibria in D. First, the worms free equilibrium, $W E \equiv\left(\frac{b}{\mu}, 0,0\right) \quad$ and $\quad$ second, an endemic equilibrium, $E E \equiv\left(S^{*}, I^{*}, P^{*}\right) \equiv\left(\frac{\theta}{\beta}, \frac{b \beta-\mu \theta}{\theta(\beta+u \theta)}, \frac{b \beta-\mu \theta}{(\beta+u \theta)}\right) \quad$ where, $\mu+\eta+\gamma=\theta \quad$ and $\quad$ endemic equilibrium belongs to the interior of $\mathrm{D}$, i.e., $\bar{D}$ with $R_{0}$ the basic reproduction number defined by, $R_{0}=\frac{b \beta}{\mu+\eta+\gamma}$ and EE exists in D and it is unique, if and only if, $R_{0}>1$.

The Jacobian matrix of model (6) at the worm free equilibrium point $W E$ is given as

$$
J(W E) \equiv\left(\begin{array}{ccc}
-\mu & 0 & -\frac{b u}{\mu} \\
0 & -(\mu+\eta+\gamma) & 0 \\
0 & 0 & -\frac{1}{T}
\end{array}\right)
$$

Its eigen values $-\mu,-(\mu+\eta+\gamma)$ and $-1 / \mathrm{T}$ are negative. Therefore, the equilibrium point $W E$ is locally asymptotically stable.

Theorem 1(a) If $R_{0} \leq 1$, then $W E \equiv\left(\frac{b}{\mu}, 0,0\right)$ is the only equilibrium and it is globally stable in $D$

(b) If $R_{0}>1$, then WE is unstable and there exists a unique endemic equilibrium EE. Furthermore, all solutions starting in D and sufficiently close to WE move away from WE. 
Proof: (a) We define Liapunov's function $\quad L=(\beta+\mu) I$

If $\mathrm{R}_{0} \leq 1$, then $\quad \mathrm{L}^{\prime}=(\beta+\mu)[\beta \mathrm{S}-(\mu+\eta+\gamma)] \mathrm{I} \leq 0$

Furthermore, $\mathrm{L}^{\prime}=0$ if and only if $\mathrm{I}=0$ or $\mathrm{R}_{0}=1$ and $\mathrm{S}=1$. Therefore, the largest compact invariant set in $\left\{(\mathrm{S}, \mathrm{I}, \mathrm{P}) \in D: \mathrm{L}^{\prime}=0\right\}$ is the singleton $\{\mathrm{WE}\}$. From Liapunov theory [12], WE is globally stable in $D$. The second part (b) follows from the fact that $\mathrm{L}^{\prime}>0$ if $\mathrm{I}>0$ and $S>\frac{1}{R_{0}}$.

Note: If $\mathrm{R}_{0}>1$, then the worms-free equilibrium is unstable by Theorem 1 . Moreover, the behavior of the local dynamics near WE as described in Theorem 1 implies that the system (6) is uniformly persistent in D, i.e., there exists a constant a $>0$, such that

$$
\begin{array}{cllll}
\lim _{t \rightarrow \infty} \inf S(t)>a & \lim _{t \rightarrow \infty} \inf I(t)>a & \lim _{t \rightarrow \infty} \inf P(t)>a \\
\lim _{t \rightarrow \infty} \inf [1-S(t)-I(t)-P(t)]>a & \text { provided } & (\mathrm{S}(0), \mathrm{I}(0), \mathrm{P}(0)) \in \mathrm{D} & {[13-15] .} & \text { Hence } a \text { is }
\end{array}
$$

independent of initial data in $\mathrm{D}$. This can be proved by applying a uniform persistent result in [14] and using a similar argument as in the proof of [16].

Theorem 2: If $\mathrm{R}_{0}>1$, then the unique endemic equilibrium 'EE' is globally stable in $\bar{D}$.

Proof: The Jacobian matrix $J=\frac{\partial f}{\partial x}$ associated with a general solution ( $\left.\mathrm{S}(\mathrm{t}), \mathrm{I}(\mathrm{t}), \mathrm{R}(\mathrm{t})\right)$ to (6) is

$$
J \equiv\left\{\begin{array}{ccc}
-(\beta I+\mu+u P) & -\beta S & -u S \\
\beta I & \beta S-(\mu+\eta+\gamma) & 0 \\
\frac{\beta I}{T} & \frac{\beta S}{T} & -\frac{1}{T}
\end{array}\right)
$$

Its second compound additive matrix $\mathbf{J}^{[2]}$ is given as

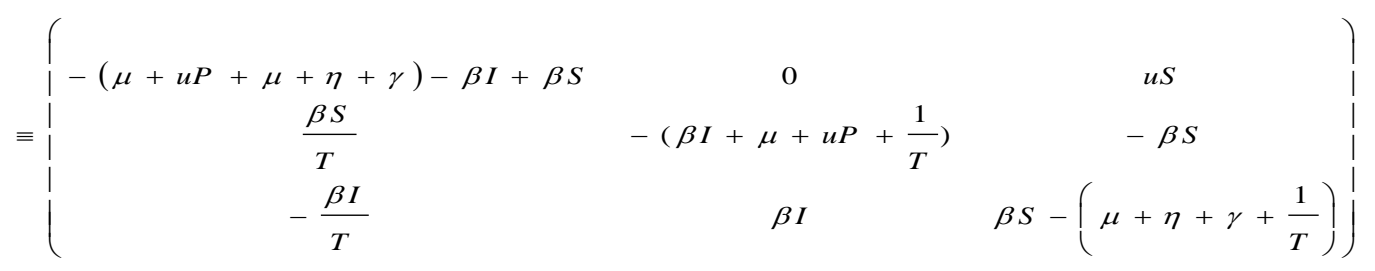

Now, set the function $P(\mathrm{x})=P(\mathrm{~S}, \mathrm{I}, \mathrm{P})$ as $\quad P(\mathrm{~S}, \mathrm{I}, \mathrm{P})=\operatorname{diag}\left[\frac{S}{I}, \frac{S}{I}, \frac{S}{I}\right\rceil$ 
Therefore, $P_{\mathrm{f}} P^{-1}=\operatorname{diag}\left\lceil\frac{\dot{S}}{S}-\frac{\dot{I}}{I}, \frac{\dot{S}}{S}-\frac{\dot{I}}{I}, \frac{\dot{S}}{S}-\frac{\dot{I}}{I}\right\rceil \quad$ Also, $P \mathrm{~J}^{[2]} P^{-1}=\mathrm{J}^{[2]}$

Therefore, $\quad \mathrm{B}=\boldsymbol{P}_{\mathrm{f}} \boldsymbol{P}^{-1}+\boldsymbol{P} \mathrm{J}^{[2]} \mathrm{P}^{-1}=\left(\begin{array}{ll}B_{11} & B_{12} \\ B_{21} & B_{22}\end{array}\right)$

where, $B_{11}=\frac{\dot{S}}{S}-\frac{\dot{I}}{I}-2 \mu-u P-\eta-\gamma-\beta I+\beta S \quad ; \quad B_{12}=\left(\begin{array}{ll}0 & u S\end{array}\right) \quad$;

$$
\begin{aligned}
B_{21}=\left(\begin{array}{ll}
\frac{\beta S}{T} & \left.-\frac{\beta I}{T}\right)^{T} \\
B_{22}= & \left(\begin{array}{c}
\frac{\dot{S}}{S}-\frac{\dot{I}}{I}-\left(\beta I+\mu+u P+\frac{1}{T}\right) \\
\beta I
\end{array}\right) \\
\frac{\dot{S}}{S}-\frac{\dot{I}}{I}+\beta S-\left(\mu+\eta+\gamma+\frac{1}{T}\right)
\end{array}\right) \mid
\end{aligned}
$$

We consider the norm in $\mathrm{R}^{3}$ as $\|(\mathrm{x}, \mathrm{y}, \mathrm{z})\|=\max \{|\mathrm{x}|,|\mathrm{y}|+|\mathrm{z}|\}$, where $(\mathrm{x}, \mathrm{y}, \mathrm{z})$ denotes a vector in $\mathrm{R}^{3}$ and we denote by $\sigma$, the Lozinskii measure with respect to this norm. We have the estimate,

$$
\sigma(B) \leq \sup \left\{g_{1}, g_{2}\right\}
$$

where $\mathrm{g}_{1}=\sigma_{1}\left(\mathrm{~B}_{11}\right)+\left|\mathrm{B}_{12}\right| ; \quad \mathrm{g}_{2}=\sigma_{1}\left(\mathrm{~B}_{22}\right)+\left|\mathrm{B}_{21}\right|$ and $\left|\mathrm{B}_{12}\right|,\left|\mathrm{B}_{21}\right|$ are matrix norms with respect to the $l_{l}$ vector norm and $\sigma_{1}$ denotes the Lozinskii measure with respect to the $l_{l}$ norm[17--20].

So, we have $\sigma_{1}\left(B_{11}\right)=\frac{\dot{S}}{S}-\frac{\dot{I}}{I}-2 \mu-u P-\eta-\gamma-\beta I+\beta S$

$$
\left|\mathrm{B}_{12}\right|=\mathrm{uS} \quad ;\left|B_{21}\right|=\frac{\beta S}{T}+\frac{\beta I}{T}
$$

To calculate $\sigma_{1}\left(\mathrm{~B}_{22}\right)$, we add the absolute values of the off-diagonal elements to the diagonal one in each column of $\mathrm{B}_{22}$ and then take the maximum of the two sums and so we get

$$
\sigma_{1}\left(B_{22}\right)=\frac{\dot{S}}{S}-\frac{\dot{I}}{I}-\frac{1}{T}+\max \{-\mu-u P, 2 \beta S-\mu-\eta-\gamma\}
$$

Therefore, $g_{1}=\frac{\dot{S}}{S}-\beta I-\mu-u P+u S$ 
$g_{2}=\frac{\dot{S}}{S}+\max \left\{-\beta S+\eta+\gamma-\frac{1}{T}+\frac{\beta S}{T}+\frac{\beta I}{T}-u P, \beta S-\frac{1}{T}+\frac{\beta S}{T}+\frac{\beta I}{T}\right\}$

Hence, $\sigma(B) \leq \sup \left\{g_{1}, g_{2}\right\}$

$\leq \frac{\dot{S}}{S}+\max \left\{u S-\mu-u P-\beta I, \eta+\gamma+\frac{\beta S}{T}+\frac{\beta I}{T}-\beta S-u P-\frac{1}{T}, \beta S-\frac{1}{T}+\frac{\beta S}{T}+\frac{\beta I}{T}\right\}$

We consider ' $a$ ' as a constant of uniform persistence, and so we get

$$
\sigma(B) \leq \frac{\dot{S}}{S}+\max \left\{-\mu-\beta a, \eta+\gamma+\frac{2 \beta a}{T}-\beta a-u a-\frac{1}{T}, \beta a-\frac{1}{T}+\frac{2 \beta a}{T}\right\}
$$

which can be written as

$\sigma(B) \leq \frac{\dot{S}}{S}-\min \left\{\mu+\beta a, \beta a+u a+\frac{1}{T}-\eta-\gamma-\frac{2 \beta a}{T}, \frac{1}{T}-\beta a-\frac{2 \beta a}{T}\right\}$

Now, taking the minimum term $\omega$ as

$\omega=\min \left\{\mu+\beta a, \beta a+u a+\frac{1}{T}-\eta-\gamma-\frac{2 \beta a}{T}, \frac{1}{T}-\beta a-\frac{2 \beta a}{T}\right\}$

and consider the following assumptions: $\mathrm{S}^{*}>\mathrm{a}, \frac{2 \beta a-1}{T}<-\beta a<\mu+u a$

Thus, $\sigma(B) \leq \frac{\dot{S}}{S}-\omega \quad$, where $\omega>0$.

This gives $\frac{1}{t} \int_{0}^{t} \sigma(B) d s \leq \frac{1}{t} \log \frac{S(t)}{S(0)}-\omega$

and so $\lim _{t \rightarrow \infty} \sup \sup \frac{1}{t} \int_{0}^{t} \sigma(B) d s<-\omega<0$

Hence it is shown that the criterion $\lim _{t \rightarrow \infty} \sup \sup \frac{1}{t} \int_{0}^{t} \sigma(B) d s<-\omega<0$ is satisfied and thus the endemic equilibrium is globally stable. This condition also itself proves the local stability of the endemic equilibrium [20]. 


\subsection{Worm's Optimal Control}

The main objective of our work is to minimize the attack of worms in the sensor nodes, to minimize the susceptible and infected number of sensor nodes and to maximize the total number of recovered sensor nodes by providing proper optimal control countermeasure. So, to achieve our objectives we minimize the performance measure $\mathrm{J}(\mathrm{u})$

i.e; $J(u)=\int_{0}^{T}\left[A_{1} S(t)+A_{2} I(t)+A_{3} P(t)+\frac{1}{2} r u^{2}(t)\right] d t$

subject to

$$
\begin{aligned}
\frac{d S}{d t}=b-\beta S I-\mu S-u P S & ; & \frac{d I}{d t}=\beta S I-(\mu+\eta+\gamma) I \\
\frac{d R}{d t}=\gamma I-\mu R+u P S \quad & & \\
\frac{d P}{d t}=\frac{1}{T}(\beta S I-P) & &
\end{aligned}
$$

where we consider, a control variable $\mathrm{u}(\mathrm{t}) \in \mathrm{U}$ which is the percentage of susceptible sensor nodes being protected per unit time [21].

Here $\mathrm{U}=\left\{\mathrm{u} / \mathrm{u}(\mathrm{t})\right.$ is measurable, $\left.0 \leq \mathrm{u}(\mathrm{t}) \leq 1, \mathrm{t} \in\left[0, \mathrm{~T}^{\prime}\right]\right\}$ shows an admissible control and $A_{1}, A_{2}, A_{3}$ are small positive constants that maintain balance in the size of $S(t), I(t), P(t)$ respectively. The square of control variable decrease the severity of the side effects of the shelter to the sensor nodes and $\mathrm{r}$ is a positive weight.

Then we rewrite the model (9) in the Matrix form:

$\phi_{\mathrm{t}}=\mathrm{B}(\phi)+\mathrm{F}(\phi)$

where, $\left.\phi=\left(\begin{array}{l}S(t) \\ I(t) \\ R(t) \\ c(t) \\ P(t)\end{array}\right), B=\left(\begin{array}{ccccc}-\mu & 0 & 0 & 0 & 0 \\ 0 & -(\mu+\eta+\gamma) & 0 & 0 & 0 \\ 0 & \gamma & -\mu & 0 & 0 \\ \mu & \mu+\eta & \mu & 0 & 0 \\ 0 & 0 & 0 & 0 & -\frac{1}{T}\end{array}\right), \quad F=\mid \begin{array}{c}-\beta S I-u P S \\ \beta S I \\ u P S \\ 0 \\ \frac{\beta S I}{T}\end{array}\right)$

and $\phi_{\mathrm{t}}$ denotes the derivative of $\phi$ with respect to the time t. Equation (10) is a non-linear system with a bounded coefficient.

We set

$$
\mathrm{D}(\phi)=\mathrm{B}(\phi)+\mathrm{F}(\phi)
$$


and $\mathrm{F}(\phi)$ satisfies

$$
\begin{gathered}
\left|F\left(\phi_{1}\right)-F\left(\phi_{2}\right)\right| \leq\left(2+\frac{1}{T}\right) \frac{\beta b}{\mu}\left|I_{1}-I_{2}\right|+\left|\frac{\left(2+\frac{1}{T}\right) b \beta \mu+2 u \beta b^{2}}{\mu^{2}}\right|\left|S_{1}-S_{2}\right|+\frac{2 u b}{\mu}\left|P_{1}-P_{2}\right| \\
\leq K\left|I_{1}-I_{2}\right|+\left|S_{1}-S_{2}\right|+\left|P_{1}-P_{2}\right|
\end{gathered}
$$

where, the positive constant $K=\max \left\{\left(2+\frac{1}{T}\right) \frac{\beta b}{\mu}, \frac{\left(2+\frac{1}{T}\right) b \beta \mu+2 u \beta b^{2}}{\mu^{2}}, \frac{2 u b}{\mu}\right\}$ is

independent of the state variable $\mathrm{S}(\mathrm{t}), \mathrm{I}(\mathrm{t}) \leq \frac{b}{\mu}$ and $\mathrm{P}(\mathrm{t}) \leq \frac{\beta b^{2}}{\mu^{2}}$.

Also, we obtain $\left|D\left(\phi_{1}\right)-D\left(\phi_{2}\right)\right| \leq M\left|\phi_{1}-\phi_{2}\right| \quad$ where, $\mathrm{M}=\max \{\mathrm{K},\|B\|\}<\infty$ (13)

Thus, it follows that the function $\mathrm{D}$ is uniformly Lipschitz continuous. Therefore, from the definition of the control variable $u(t)$ and the restriction on $S(t), I(t), R(t), C(t), P(t) \geq 0$, we see that a solution of the performance measure $\mathrm{J}(\mathrm{u})$ exist [22]. To find an optimal solution, first we find the Lagrangian and Hamiltonion for the optimal control problem (8) and (9).

The Lagrangian of the problem is given by

$\mathrm{L}(\mathrm{S}, \mathrm{I}, \mathrm{P})=A_{1} S(t)+A_{2} I(t)+A_{3} P(t)+\frac{1}{2} r u^{2}(t)$

and we define the Hamiltonian $\mathrm{H}$ for the control problem as,

$$
\begin{aligned}
\mathrm{H} & =\mathrm{L}(\mathrm{S}, \mathrm{I}, \mathrm{P})+\lambda_{1}(t) \frac{d S}{d t}+\lambda_{2}(t) \frac{d I}{d t}+\lambda_{3}(t) \frac{d R}{d t}+\lambda_{4}(t) \frac{d C}{d t}+\lambda_{5}(t) \frac{d P}{d t} \\
& =A_{1} S+A_{2} I+A_{3} P+\frac{1}{2} r u^{2}(t)+\lambda_{1}(t)\{b-\beta S I-\mu S-u P S\} \\
& +\lambda_{2}(t)\{\beta S I-(\mu+\eta+\gamma) I\}+\lambda_{3}(t)\{\gamma I-\mu R+u P S\} \\
& +\lambda_{4}(t)\{\mu(S+R)+(\mu+\eta) I\}+\lambda_{5}(t)\left\{\frac{1}{T}(\beta S I-P)\right\}
\end{aligned}
$$

where, $\lambda_{1}(t), \lambda_{2}(t), \lambda_{3}(t), \lambda_{4}(t), \lambda_{5}(t)$ are the adjoint functions, and the adjoint equations for $\lambda_{1}(t), \lambda_{2}(t), \lambda_{3}(t), \lambda_{4}(t), \lambda_{5}(t)$ are given by

$$
\frac{d \lambda_{1}}{d t}=-\frac{\partial H}{\partial S}=\lambda_{1}(\beta I+\mu+u P)-\lambda_{2} \beta I-\lambda_{3} u P-\lambda_{4} \mu-\lambda_{5} \frac{\beta I}{T}-A_{1}
$$




$$
\begin{aligned}
& \frac{d \lambda_{2}}{d t}=-\frac{\partial H}{\partial I}=\lambda_{1} \beta S-\lambda_{2}\{\beta S-(\mu+\eta+\gamma)\}-\lambda_{3} \gamma-\lambda_{4}(\mu+\eta)-\lambda_{5} \frac{\beta S}{T}-A_{2} \\
& \frac{d \lambda_{3}}{d t}=-\frac{\partial H}{\partial R}=\left(\lambda_{3}-\lambda_{4}\right) \mu \\
& \frac{d \lambda_{4}}{d t}=-\frac{\partial H}{\partial C}=0 \\
& \frac{d \lambda_{5}}{d t}=-\frac{\partial H}{\partial P}=\left(\lambda_{1}-\lambda_{3}\right) u S+\frac{\lambda_{5}}{T}-A_{3}
\end{aligned}
$$

along with the transversality conditions: $\lambda_{\mathrm{i}}\left(\mathrm{T}^{\prime}\right)=0 ; \mathrm{i}=1,2,3,4,5$

By optimality conditions, we have

$\frac{\partial H}{\partial u}=r u *(t)-\lambda_{1}(t) P * S *+\lambda_{3}(t) P * S *=0 \quad$ and $\quad$ at $\quad \mathrm{u}^{*}, \quad$ we can obtain $u *(t)=\frac{\mathrm{S} * \mathrm{P} *\left(\lambda_{1}(t)-\lambda_{3}(t)\right)}{r}$ where, $\mathrm{S} *(\mathrm{t}), \mathrm{I} *(\mathrm{t}), \mathrm{R} *(\mathrm{t}), \mathrm{C} *(\mathrm{t}), \mathrm{P} *(\mathrm{t})$ are optimal state solutions associated with optimal control variable $\mathrm{u}^{*}(\mathrm{t})$ for the performance measure problem (8) and (9). By using the property of control space, we obtain

$$
u *(t)=\max \left\{\min \left\{\frac{\left(\lambda_{1}(t)-\lambda_{3}(t)\right) S * P *}{r}, 1\right\}, 0\right\}
$$

Now, the optimal points can be obtained after solving the following system of equations

$$
\begin{array}{ll}
\frac{d S *}{d t}=b-\beta S * I *-\mu S *-u *(t) P * S * & \frac{d I *}{d t}=\beta S * I *-(\mu+\eta+\gamma) I * \\
\frac{d R *}{d t}=\gamma I *-\mu R *+u *(t) P * S * & \quad ; \quad \frac{d C *}{d t}=\mu(S *+R *)+(\mu+\eta) I * \\
\frac{d P *}{d t}=\frac{1}{T}(\beta S * I *-P *) & \text { (18) }
\end{array}
$$

with the Hamiltonion 


$$
\begin{aligned}
& H *=A_{1} S *+A_{2} I *+A_{3} P *+\frac{1}{2} r\left\{\max \left\{\min \left\{\frac{\left(\lambda_{1}(t)-\lambda_{3}(t)\right) S * P *}{r}, 1\right\}, 0\right\}\right\}^{2} \\
& +\lambda_{1}(t)\left\{b-\beta S * I *-\mu S *-\left\{\max \left\{\min \left\{\frac{\left(\lambda_{1}(t)-\lambda_{3}(t)\right) S * P *}{r}, 1\right\}, 0\right\}\right\} P * S *\right\} \\
& +\lambda_{2}(t)\{\beta S * I *-(\mu+\eta+\gamma) I *\} \\
& +\lambda_{3}(t)\left\{\gamma I *-\mu R *+\left\{\max \left\{\min \left\{\frac{\left(\lambda_{1}(t)-\lambda_{3}(t)\right) S * P *}{r}, 1\right\}, 0\right\}\right\} P^{*} S *\right\} \\
& +\lambda_{4}(t)\{\mu(S *+R *)+(\mu+\eta) I *\}+\lambda_{5}(t)\left\{\frac{1}{T}(\beta S * I *-P *)\right\}
\end{aligned}
$$

\subsection{Variations of the Model with Additional Control Measures}

\subsubsection{SIRC with Quarantine (SIQRC Model)}

One intervention procedure to physically control the spread of a malicious attack is by quarantining of infectious nodes. This can be structurally modeled by introducing a new compartment known as the Quarantined class $\mathrm{Q}(\mathrm{t})$ in which some most infectious sensor nodes are forcibly isolated from the sensor field in order to reduce maximum transmission of the malicious signals to susceptible class of sensor nodes. The effect of quarantining is often seen as a functional measure to reduce the rate at which new susceptible nodes get infected and also to have a faster rate of recovery of infectious nodes. The major impact is however in the reduction of the rate of formation of newer infective nodes. The modified model can be represented by the following system of equations

$$
\begin{array}{lll}
\frac{d S}{d t}=b-\beta S I-\mu S-u P S & ; & \frac{d I}{d t}=\beta S I-(\mu+\eta+\gamma+\delta) I \\
\frac{d Q}{d t}=\delta I-(\mu+\eta+\varepsilon) Q & ; & \frac{d R}{d t}=\gamma I+\varepsilon Q-\mu R+u P S \\
\frac{d C}{d t}=\mu S+\mu R+(\mu+\eta)(I+Q) ; & \frac{d P}{d t}=\frac{1}{T}(\beta S I+\delta I-P)
\end{array}
$$

In this model we have considered $g(S, I, Q)=\beta S I+\delta I$

For this system the worm free equilibrium is observed to be $\operatorname{WE}_{1}\left(\frac{b}{\mu}, 0,0,0\right)$ and the $\begin{array}{lllll}\text { endemic } & \text { equilibrium } & \text { point } & \text { is } & \mathrm{EE}_{1}\end{array}$ $\left(S_{1}, I_{1}, Q_{1}, P_{1}\right) \equiv\left(\frac{\phi}{\beta}, \frac{b \beta-\mu \phi}{\phi(\beta+u \psi)}, \frac{\delta(b \beta-\mu \phi)}{\phi(\mu+\eta+\varepsilon)(\beta+u \psi)}, \frac{\psi(b \beta-\mu \phi)}{\phi(\beta+u \psi)}\right)$ where, $(\mu+\eta+\gamma+\delta)=\phi ; \quad(\mu+\eta+\gamma+2 \delta)=\psi$.

Also the basic reproduction number is $R_{0}=\frac{\beta}{(\mu+\eta+\gamma+\delta)}$

At the worm free equilibrium point $\mathrm{WE}_{1}$, the Jacobian matrix of the system is 


$$
J\left(W E_{1}\right)=\left(\begin{array}{cccc}
-\mu & 0 & 0 & -\frac{u b}{\mu} \\
0 & -(\mu+\eta+\gamma+\delta) & 0 & 0 \\
0 & \delta & -(\mu+\eta+\varepsilon) & 0 \\
0 & \frac{\delta}{T} & 0 & -\frac{1}{T}
\end{array}\right)
$$

Its eigen values are: $-\mu,-(\mu+\eta+\gamma+\delta),-(\mu+\eta+\varepsilon)$, and $-\frac{1}{T}$, which all are negative; hence the system is locally asymptotically stable at the point $\mathrm{WE}_{1}$.

To show that the endemic equilibrium point $E E_{l}$ is locally asymptotically stable, when $\mathrm{R}_{\mathrm{o}}>$ 1 we have the characteristics equation of the linearization of system near $E E_{l}$ as

$$
\lambda^{4}+a_{1} \lambda^{3}+a_{2} \lambda^{2}+a_{3} \lambda+a_{4}=0
$$

where, $a_{1}=\mu+\eta+\varepsilon+\frac{1}{T}+\beta I_{1}+\mu+u P_{1}>0$

$$
\begin{aligned}
& a_{2}=(\mu+\eta+\varepsilon) \frac{1}{T}+\left(\beta I_{1}+\mu+u P_{1}\right)\left(\mu+\eta+\varepsilon+\frac{1}{T}\right)+\beta^{2} S_{1} I_{1}+\frac{\beta u S_{1} I_{1}}{T}>0 \\
& a_{3}=(\mu+\eta+\varepsilon) \frac{1}{T}\left(\beta I_{1}+\mu+u P_{1}\right)+\beta^{2} S_{1} I_{1}\left(\mu+\eta+\varepsilon+\frac{1}{T}\right)+\beta u S_{1} I_{1}\left(\frac{\beta S_{1}+\delta}{T}\right)+\frac{\beta u S_{1} I_{1}}{T}(\mu+\eta+\varepsilon)>0 \\
& a_{4}=\frac{\beta^{2} S_{1} I_{1}}{T}(\mu+\eta+\varepsilon)+\beta u S_{1} I_{1}\left(\frac{\beta S_{1}+\delta}{T}\right)(\mu+\eta+\varepsilon)>0
\end{aligned}
$$

Also we have $a_{1} a_{2} a_{3}-a_{3}^{2}-a_{1}^{2} a_{4}>0$ if $\mathrm{T}>0$, thus endemic equilibrium point is locally asymptotically stable.

\subsubsection{SIRC with Vaccination (SIRVC Model)}

In this case instead of using quarantining as a method for reducing the spread of infection, we use the method of vaccination. As in biological vaccination, here also a number of non infected susceptible sensor nodes are directly immunized from the effect of the worm. This measure prevents the sensor nodes from being infected for a certain period of time, after which the nodes tend to lose their immunity to attack and so again become susceptible. In the structure of the model, a new compartment $\mathrm{V}$ is added which represents the sensor nodes that are vaccinated at a given instant of time. The parameters $\mathrm{p}$ and $\mathrm{q}$ are respectively the rates at which susceptible nodes are vaccinated and at which vaccinated nodes lose their immunity and again become susceptible. The system of equations now gets modified to

$$
\begin{aligned}
\frac{d S}{d t}=b-\beta S I-\mu S-p S-u P S+q V ; & & \frac{d I}{d t}=\beta S I-(\mu+\eta+\gamma) I \\
\frac{d R}{d t}=\gamma I-\mu R+u P S & ; & \frac{d V}{d t}=p S-q V-\mu V
\end{aligned}
$$


$\frac{d C}{d t}=\mu(S+R+V)+(\mu+\eta) I \quad ; \quad \frac{d P}{d t}=\frac{1}{T}(\beta S I-P)$

In this model we have considered $\mathrm{g}(\mathrm{S}, \mathrm{I})=\beta \mathrm{SI}$

For this system the worm free equilibrium is observed to be $\mathrm{WE}_{2}$ $\left(\frac{b(\mu+q)}{\mu(\mu+p+q)}, 0,0, \frac{p b}{\mu(\mu+q+p)}\right) \quad$ and the endemic equilibrium point is $\mathrm{EE}_{2}$ $\left(S_{2}, I_{2}, V_{2}, P_{2}\right)$

$\equiv\left(\frac{\mu+\eta+\gamma}{\beta}, \frac{b \beta(\mu+q)+\xi(q p-\mu-p)}{\xi(\mu+q)(\beta+u \xi)}, \frac{p(\mu+\eta+\gamma)}{\beta(\mu+q)}, \frac{\xi(b \beta(\mu+q)+\xi(q p-\mu-p))}{\xi(\mu+q)(\beta+u \xi)}\right)$

and the basic reproduction number is defined as $R_{o}=\frac{b \beta}{(\mu+\eta+\gamma)}$

At worm free equilibrium point $\mathrm{WE}_{2}$, the Jacobian matrix of system is

$$
J\left(W E_{2}\right)=\left(\begin{array}{cccc}
-(\mu+p) & 0 & q & -\frac{u b(\mu+q)}{\mu(\mu+p+q)} \\
0 & -(\mu+\eta+\gamma) & 0 & 0 \\
p & 0 & -(\mu+q) & 0 \\
0 & 0 & 0 & -\frac{1}{T}
\end{array}\right)
$$

Its eigen values are: $-(\mu+p),-(\mu+\eta+\gamma),-(\mu+q)$, and $-\frac{1}{T}$

which are all negative; hence the system is locally asymptotically stable at $\mathrm{WE}_{2}$ 1

To show that the endemic equilibrium point $E E_{2}$ is locally asymptotically stable, when $\mathrm{R}_{\mathrm{o}}>$ we have the characteristics equation of the linearization of system near $E E_{2}$ as

$$
\lambda^{4}+a_{1} \lambda^{3}+a_{2} \lambda^{2}+a_{3} \lambda+a_{4}=0
$$

where, $a_{1}=2 \mu+\beta I_{2}+p+q+u P_{2}+\frac{1}{T}>0$

$$
\begin{aligned}
& a_{2}=\left(\beta I_{2}+\mu+p+u P_{2}\right)(\mu+q)+\frac{\beta I_{2}+2 \mu+p+u P_{2}+q}{T}+\beta^{2} S_{2} I_{2}-p q+\frac{\beta u S_{2} I_{2}}{T}>0 \\
& a_{3}=(\mu+q) \frac{1}{T}\left(\beta I_{2}+\mu+p+u P_{2}\right)+\beta^{2} S_{2} I_{2}\left(\mu+q+\frac{1}{T}\right)-\frac{p q}{T}+\frac{\beta^{2}}{T} u\left(S_{2}\right)^{2} I_{2}+\frac{\beta u S_{2} I_{2}}{T}(\mu+q)>0 \\
& a_{4}=\frac{\beta^{2} S_{2} I_{2}}{T}(\mu+q)+\frac{\beta^{2}}{T} u\left(S_{2}\right)^{2} I_{2}(\mu+q)>0
\end{aligned}
$$


And so simply we have $a_{1} a_{2} a_{3}-a_{3}^{2}-a_{1}^{2} a_{4}>0$ if $\mathrm{T}>0$, thus endemic equilibrium point is locally asymptotically stable.

\section{Numerical Method and Analysis}

Runge - Kutta- Fehlberg method of order 4 and 5 is employed to solve and simulate the systems (5) with the help of MATLAB. Functionally, the models show two basic dimensions: firstly, the stability with respect to the equilibrium points, which depends on the threshold condition given by $\mathrm{R}_{\mathrm{o}}$ and secondly the optimal control countermeasure, which allows us to minimize the number of infected sensor nodes and to maximize the number of recovered nodes. Figure 3 shows the overall dynamics of the SIRC model, where the asymptotic behavior of the classes can be clearly observed. Figure 4 to 6 shows the variation of the recovered class corresponding to the infectious class for the SIRC model and its two variations. In figure 7 the scale of infection has been shown with respect to time, where the impact of the optimal control countermeasure on the magnitude of the infectious population is clearly visible. Figure 8 and figure 9 show the asymptotic stability of the worm free equilibrium and the endemic equilibrium for the values of the basic reproduction number given by $R_{0} \leq 1$ and $R_{0}>1$ respectively. Figure 10 shows the variations is the recovered class with respect to the susceptible class. Figure 11 exhibits the stability of the worm free equilibrium in the SIQRC model when $R_{0} \leq 1$. Figure 12 shows the relative level of the infection reached in the SIRC model and when quarantining and vaccination of nodes are used as control measures.

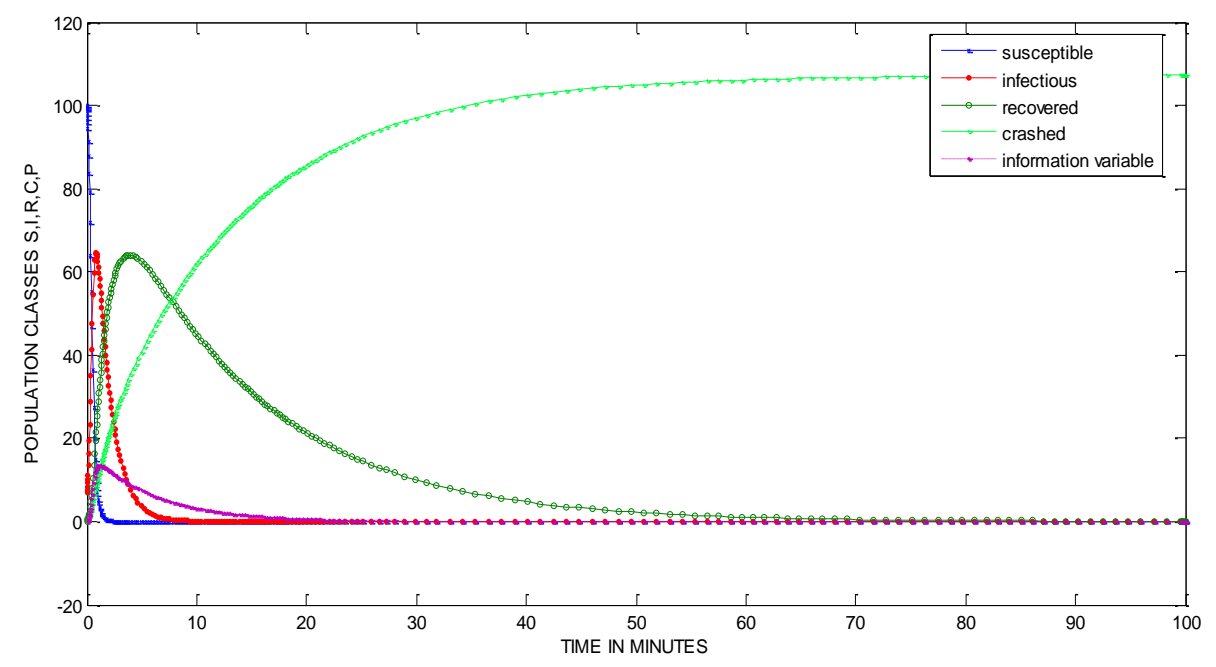

Figure 3. Global Dynamics of SIRC Model of System (5) with Parameters $b=0.004 ; \mu=0.075 ; u=0.009 ; \beta=0.06 ; \eta=0.075 ; \gamma=0.6 ; T=6$ 


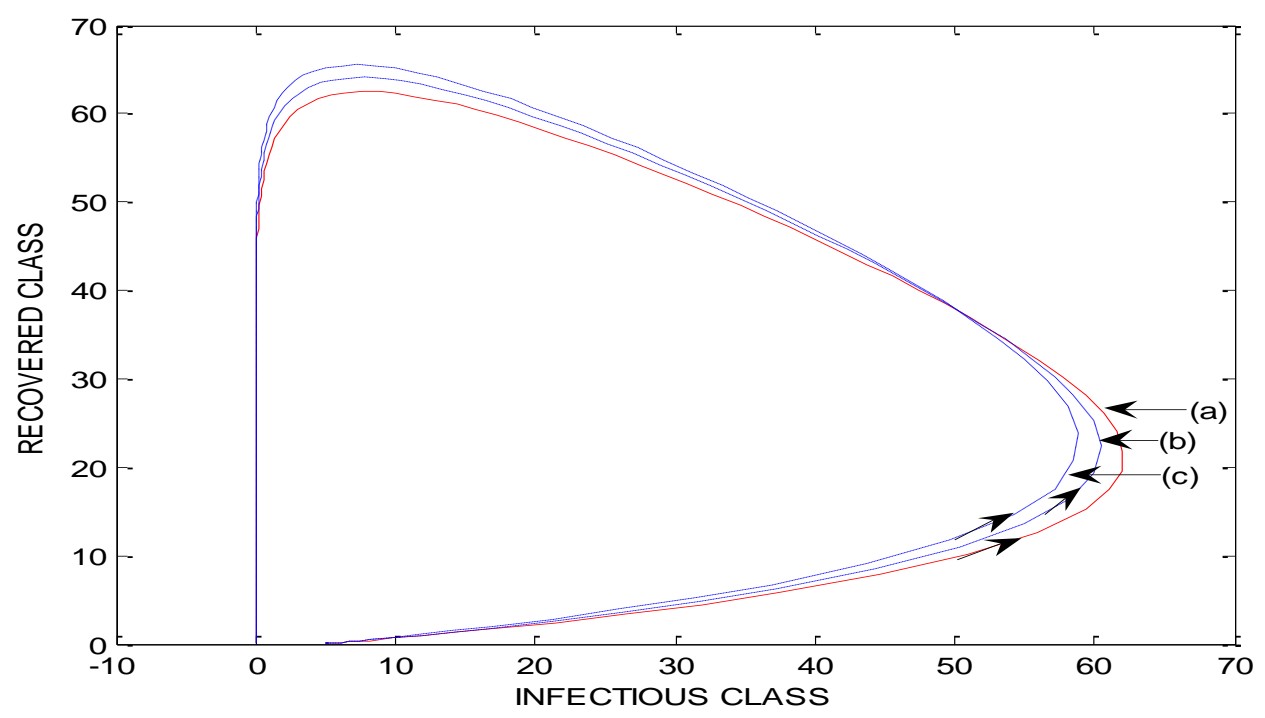

Figure 4. Variation of Recovered Class Verses Infectious Class for SIRC Model with Parameters (a) $b=0.004 ; \mu=0.075 ; u=0.009 ; \beta=0.06 ; \eta=0.075 ; \gamma=0.6 ; T=6$; (b) $b=0.004 ; \mu=0.075 ; u=0.009 ; \beta=0.06 ; \eta=0.075 ; \gamma=0.65 ; T=6 ; \quad$ (c) $b=0.004 ;$ $\mu=0.075 ; u=0.009 ; \beta=0.06 ; \eta=0.075 ; \gamma=0.7 ; T=6$

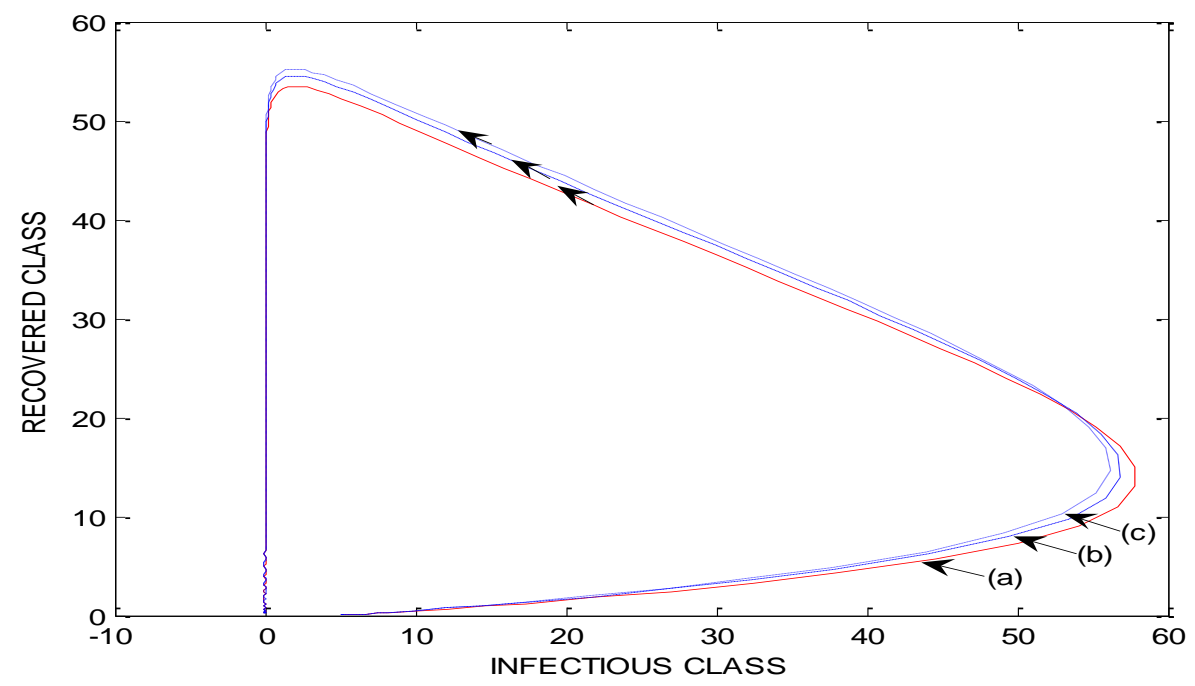

Figure 5. Variation of Recovered Class Verses Infectious Class for Model SIRC with Quarantine with Parameters (a): $b=0.001 ; \mu=0.06 ; u=0.002 ; \beta=0.05$; $\eta=0.045 ; \gamma=035 ; \delta=0.3 ; \varepsilon=0.25 ; T=5$. (b): $b=0.001 ; \mu=0.06 ; u=0.002 ; \beta=0.05$; $\eta=0.045 ; \gamma=038 ; \delta=0.3 ; \varepsilon=0.25 ; T=5$. (c): $b=0.001 ; \mu=0.06 ; u=0.002 ; \beta=0.05$; $\eta=0.045 ; \gamma=0.4 ; \delta=0.3 ; \varepsilon=0.25 ; T=5$ 


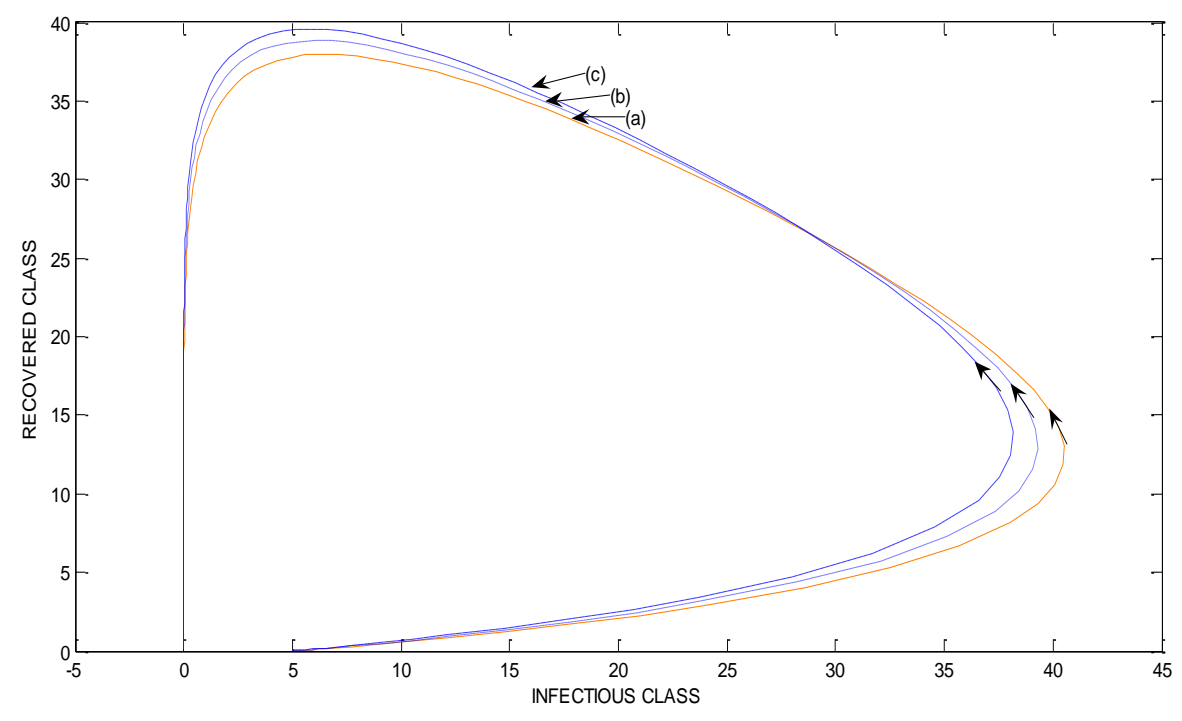

Figure 6. Variation of Infectious Class Verses Recovered Class for Model SIRC with Vaccinated with Parameters (a) $b=0.001 ; \mu=0.06 ; u=0.002 ; \beta=0.04 ; \eta=0.03$; $\gamma=0.35 ; p=0.5 ; q=0.02 ; T=6$. (b) $b=0.001 ; \mu=0.06 ; u=0.002 ; \beta=0.04 ; \eta=0.03 ; \gamma=0.38$;

$p=0.5 ; q=0.02 ; T=6$. (c) $b=0.001 ; \mu=0.06 ; u=0.002 ; \beta=0.04 ; \eta=0.03 ; \gamma=0.41$; $\mathrm{p}=0.5 ; \mathrm{q}=0.02 ; \mathrm{T}=6$

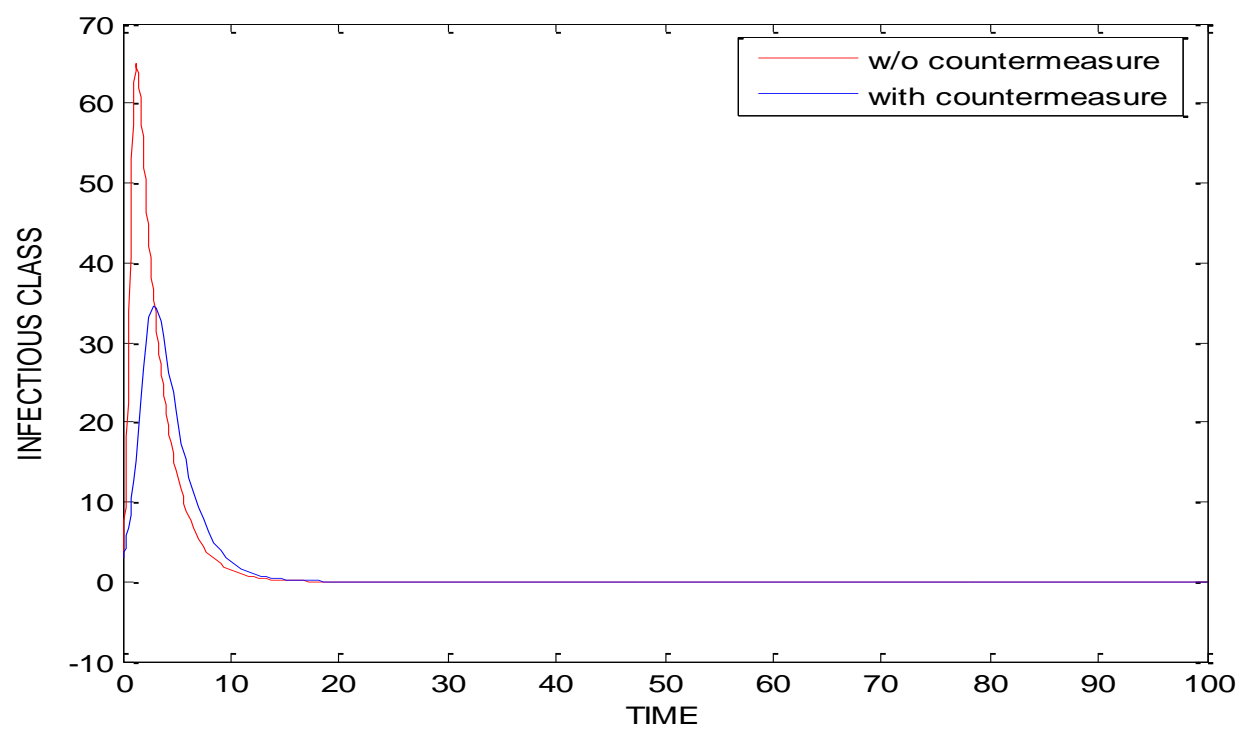

Figure 7. Impact of Presence of Optimal Countermeasure with Parameters (a) without countermeasure: $b=0.001 ; \mu=0.06 ; u=0.015 ; \beta=0.05 ; \eta=0.045 ; \gamma=0.35$; $T=5$; (b) with countermeasure: $b=0.001 ; \mu=0.06 ; u=0.015 ; \beta=0.02 ; \eta=0.045$; $\gamma=0.35 ; \mathrm{T}=5$ 


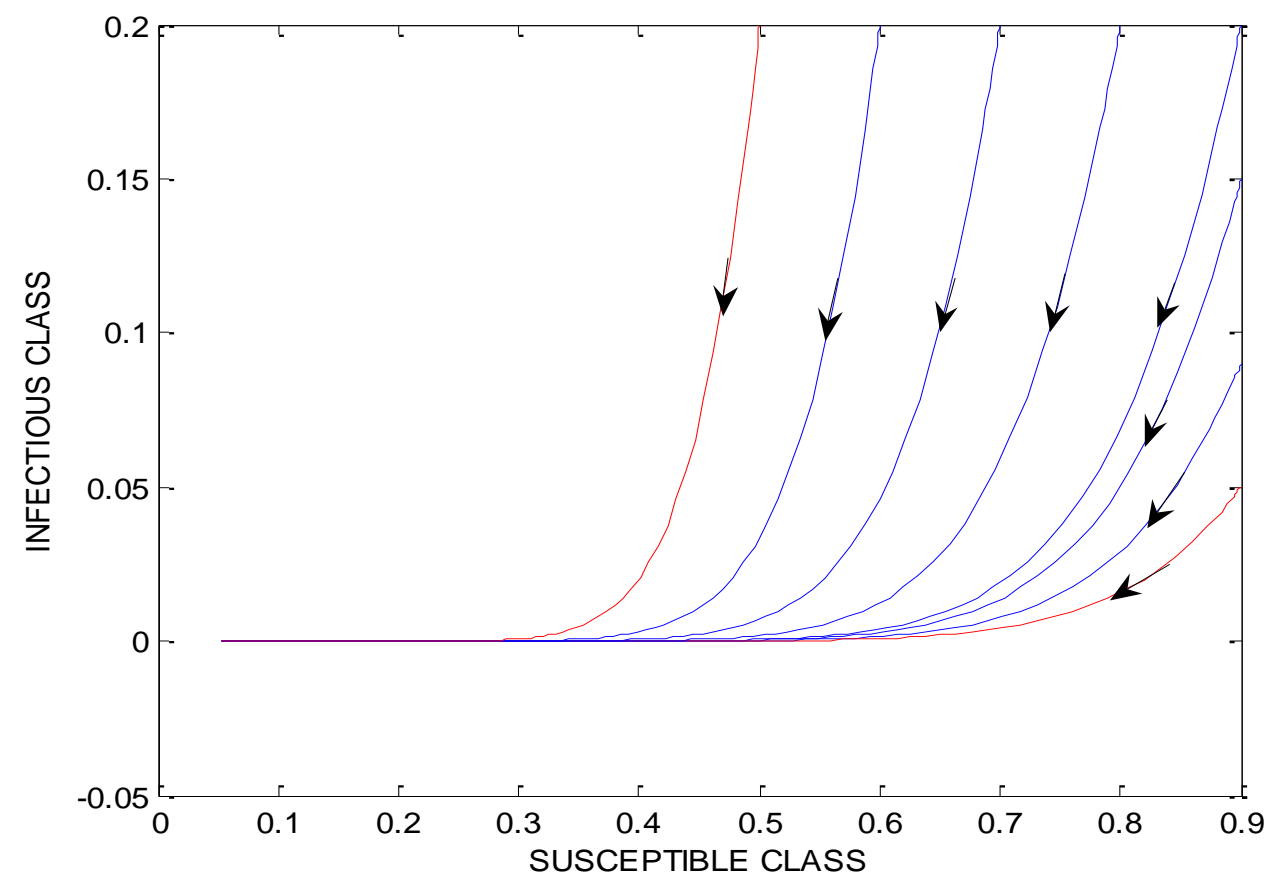

Figure 8. Existence of Worm Free Equilibrium when $\mathbf{R}_{0} \leq 1$ for SIRC Model

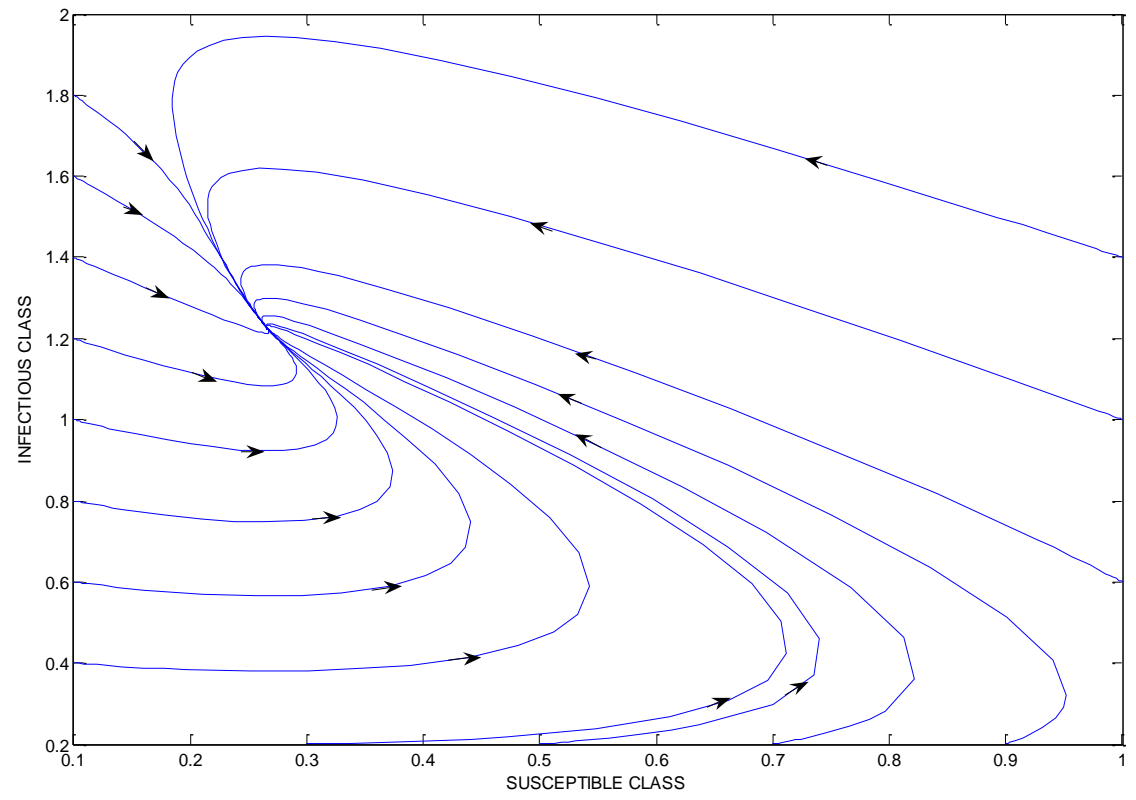

Figure 9. Existence of Endemic Equilibrium when $R_{0}>1$ for SIRC Model 


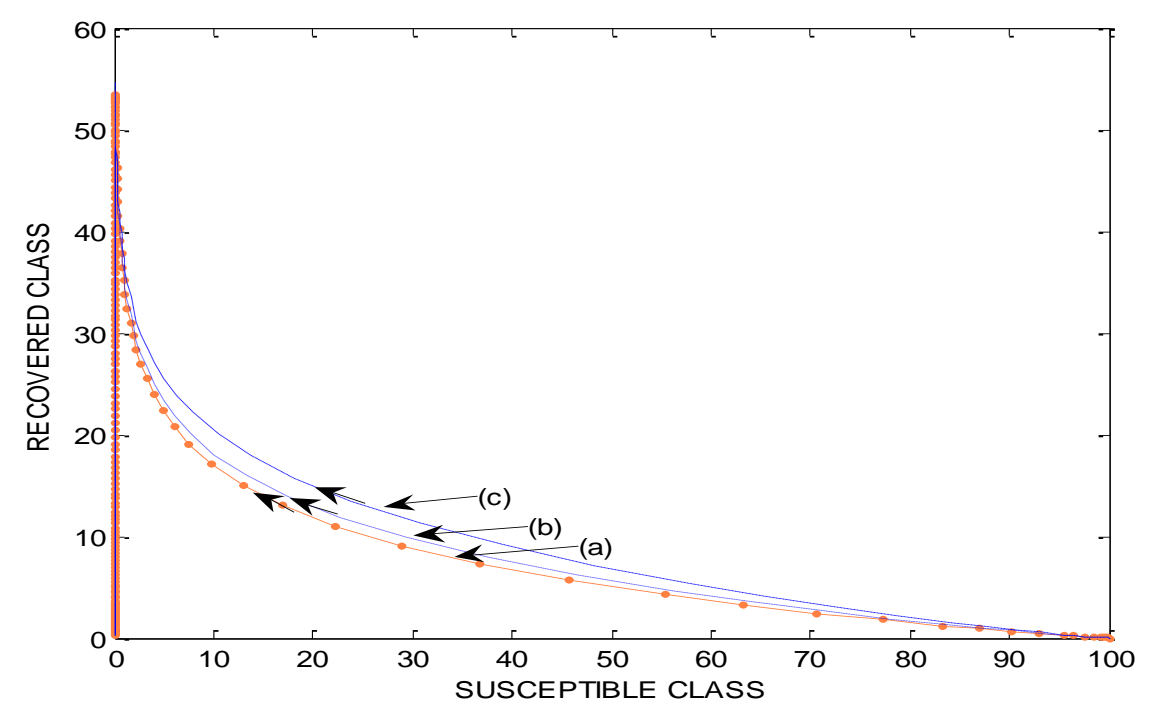

Figure 10. Variation of Recovered Class Verses Susceptible Class for SIRC Model with Quarantine with Parameters

(a): $b=0.001 ; \mu=0.06 ; u=0.002 ; \beta=0.05 ; \eta=0.045 ; \gamma=035 ; \delta=0.3 ; \varepsilon=0.25 ; T=5$.

(b): $b=0.001 ; \mu=0.06 ; u=0.009 ; \beta=0.05 ; \eta=0.045 ; \gamma=035 ; \delta=0.3 ; \varepsilon=0.25 ; T=5$.

(c): $b=0.001 ; \mu=0.06 ; u=0.015 ; \beta=0.05 ; \eta=0.045 ; \gamma=035 ; \delta=0.3 ; \varepsilon=0.25 ; T=5$.

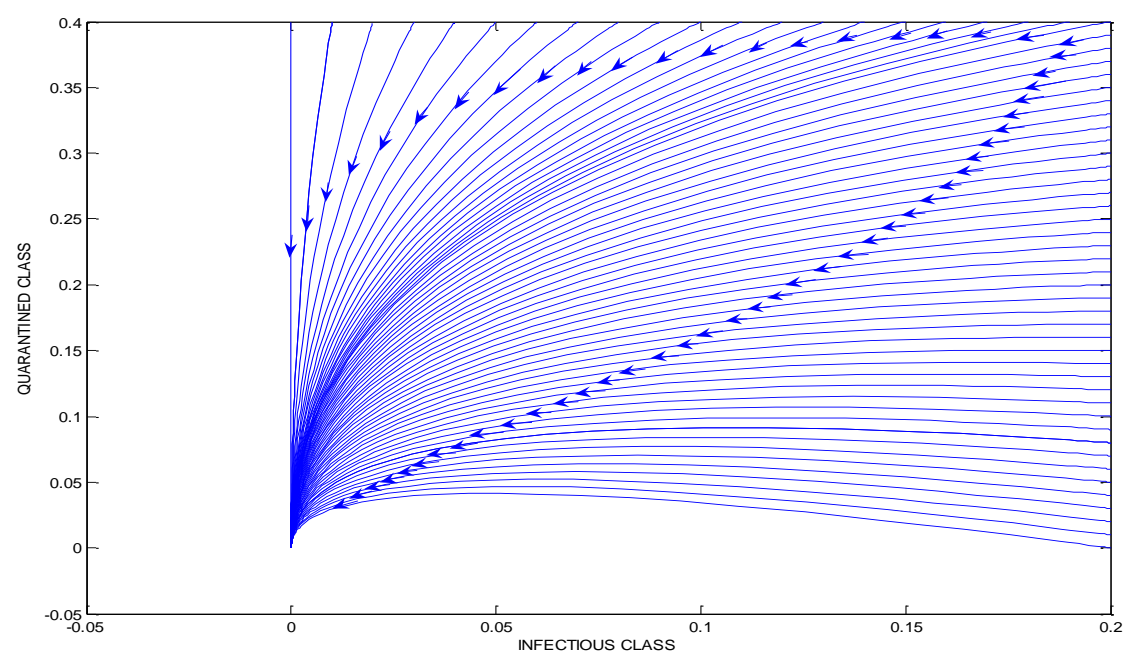

Figure 11. Existence of Worm Free Equilibrium when $R_{0} \leq 1$ for SIRC Model with Quarantine 


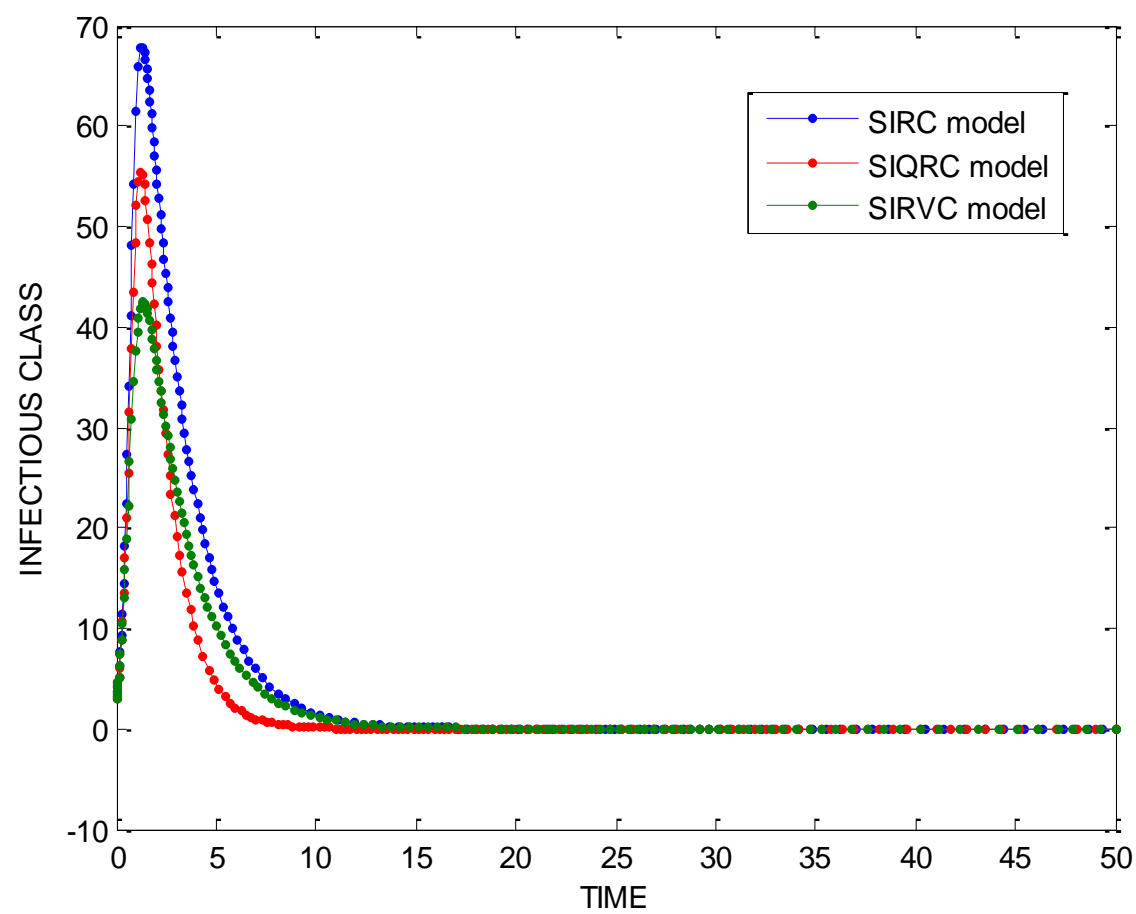

Figure 12. Variation in the Scale of the Infectious Class in the Three Models for $R_{0}<1$

\section{Conclusion}

A compartmental model: susceptible-infectious-recovered-crashed (SIRC) is developed to understand the spreading behavior of worms in WSN. A special crashed compartment is introduced that includes the nodes which crash out due to induced infection or reason other than the attack of worms. This crashed class helps us in optimizing the level of infection and also of corresponding recovery of sensor nodes in the wireless sensor network. We introduce an information variable which provides the information about both the current and past state of worm infection in WSN. A performance measure is considered which we minimize in order to maximize the total number of recovered sensor nodes by providing proper optimal control countermeasure, to minimize the attack of worms in the sensor nodes, and also to minimize the susceptible and infected number of sensor nodes. Reproduction number is obtained for the model developed and condition for existence and extinction of worms in WSN is discussed. Also the stability of the worms-free and endemic equilibrium of the epidemic model is established and it is also shown that the model may undergo a forward bifurcation. Extensive simulation is carried out to validate the models developed.

\section{References}

[1] M. H. R. Khouzani, In Fifth Inf. Theory and Appls. Workshop (ITA), University of California, San Diego, February 1-5 (2010)

[2] S. Tang and B. Mark, $7^{\text {th }}$ Int. Workshop on Des. of Reliable Commun. Netw., Oct. 25-28 (2009), pp. 86-91.

[3] E. Beretta, V. Capasso and F. Rinaldi, J. Math. Biol. 26 (1988), pp. 661- 688.

[4] X.Song and S. Cheng, J. Korean Math. Soc. 42 (5) (2005), pp. 1071-1086.

[5] B. K. Mishra and N. Jha, Appl. Math. Model. 34 (2010), pp. 710-715. 
[6] J. Ren and X. Yang, J. of Inf. \& Comput. Sci. 8: 9 (2011), pp. 1735-1745.

[7] J. K. Hale, $2^{\text {nd }}$ ed, Krieger, Basel (1980).

[8] A. d'Onofrio, P. Manfredi and E. Salinelli, Theor. Popul. Biol. 71 (2007), pp. 301-307.

[9] A. d'Onofrio, P. Manfredi and E. Salinelli, Math. Model. of Nat. Phenom., Epidemiology, 2(1) (2007), pp. 26- 43.

[10] J. Guckenheimer and P. Holmes, Springer-Verlag, New York (1983), pp. 117-156.

[11] T. K. Kar and P. K. Mondal, Nonlinear Anal. : Real World Appl., Volume 12, Issue 4 (August 2011), pp. 2058-2068.

[12] J.P. LaSalle, CBMS-NSF Regional Conf. Ser. in Appl. Math. 25, SIAM, Philadelphia, (1976).

[13] G. J. Butler and P. Waltman, Proc. Amer. Math. Soc. 96 (1986), pp. 425-430.

[14] H. I. Freedman, M. X. Tang and S. G. Ruan, J. Dyn. Differ. Equ. 6 (1994), pp. 583-600.

[15] P. Waltman in Delay Differ. Equ. and Dyn. Syst., Edited S. Busenberg and M. Martelli, Springer-Verlag, New York (1991) pp.31-40.

[16] M. Y. Li, J. R. Graef, L. C. Wang and J. Karsai, Math. Biosci. 160 (1999), pp. 191-213.

[17] R. H. Martin Jr, J. Math. Anal. Appl. 45 (1974), pp. 432-454.

[18] W. A. Coppel, Heath, Boston (1965).

[19] M. Y. Li and J. S. Muldowney, J. Differ. Equ. 106 (1994), pp. 27-39.

[20] M. Y. Li and J. S. Muldowney, SIAM J. Math. Anal. 27 (1996), pp. 1070-1083.

[21] G. Zaman, Y. H. Kang and I. H. Jung, Biosys., 93 (2008), pp. 240-249.

[22] G. C. Birkhoff, $4^{\text {th }}$ ed. John Wiley and Sons, New York (1989). 